\title{
Implementation of an Alternative High Performance Flip Flop to JK Flip Flop
}

\author{
${ }^{1}$ Ogunlere Samson, ${ }^{2}$ Ajaegbu Chigozirim, ${ }^{3}$ Kanu Richmond, ${ }^{4}$ Somefun Olawale \\ ${ }^{1234}$ Computer Science Department, \\ Babcock University, Ilishan-Remo, \\ Nigeria
}

\begin{abstract}
Computer performance is primarily affected by the processor and memory. If either one reaches its limits, the performance of the whole system degrades. As semiconductor technology advances, the performance gap between processor and memory has become one of the major issues in computer design. This situation causes a growing gap between processor and memory in the performance. Hence the design of an efficient and high performance memory element known as Flip-Flop Extension is of crucial importance in computer design. The analysis of the existing structures is necessary when the requirement of the Flip-Flop is for low-power and highspeed digital applications. This study is dedicated to the investigation of the existing conventional memory elements, the SET /RESET (SR) and JUMP-KEY (JK) Flip Flops performance and the result is used to evaluate the design of an alternative Flip Flop known as Flip Flop Extension. It is evidence from the analysis, that the JK-FF Extension at $87.5 \%$ has one gate less than the conventional SR \& JK-FFs. This is a great advantage in performance because fewer gates enhance performance in which numbers of gate/ transistors represent hardware cost.
\end{abstract}

Key Words: Conventional Flip Flop, Flip Flop Extension, Boolean Equation, K-Map, DeMorgan's Theorem.

\section{INTRODUCTION}

Flip-Flops are digital circuits with two stable, selfmaintaining states that are used as storage/ memory elements such as Random Access Memory (RAM), Caches Memory and Read Only Memory (ROM). They are also very useful in the following electronic digital devices design; Sequence Detector, Data Synchronizer, Frequency Divider, Registers (data transfer), Counters and Registers in Central Processing Unit (CPU) for data transfer. They are derived from Sequential Logic Circuits which are the main electronics circuits that make the development of computers possible. The ability of computer systems to operate without the continuous human intervention is solely achieved through sequential logic circuits, the building blocks of Flip Flops [1].

\section{MOTIVATION FOR THE STUDY}

It has been observed that computer performance is primarily affected by the processor and memory. If either one reaches its limits (which may initially be the memory), the performance of the whole system degrades. As semiconductor technology advances, the performance gap between processor - the Central Processing Unit (CPU) and main memory - the Random Access Memory (RAM) has become one of the major issues in computer design. In the past 45 years, an exponential rate of improvement has been witnessed in semiconductor technology. The processor performance increases at a rate of $60 \%$ per year while the memory performance increases just $10 \%$ per year $[2 ; 3]$. This situation causes a $50 \%$ growing gap between processor and memory in the performance as depicted in Figure 1.1. If memory fails to keep pace with the processor's constant demands, the processor stalls in a wait state, and valuable processing time is lost. This imbalance has become one major bottleneck in further improving the computer performance.

One reason memory system performance has consistently lagged processor performance is that memory systems typically consist of one or more chips that are designed and manufactured separately from the processor, and the performance of the interconnected multi-chip memory system is difficult to scale to achieve higher data-rate and lower access latency. Memory system data-rates are increasing with each new generation of memory devices at the rate of $100 \%$ every three years, and memory row cycle times are decreasing at a rate of approximately $7 \%$ per year [4]. The collective trends are increasing the ratio of row cycle times to the duration of data bursts on the data bus. This is why it is imperative to critically evaluate the existing conventional JK-FF and the need to bridge the speed gap between memory and processor by enhancing the memory speed through logical modification frameworks of the conventional JK-FF which utilizes $75 \%$ out of the $100 \%$ of its' states.

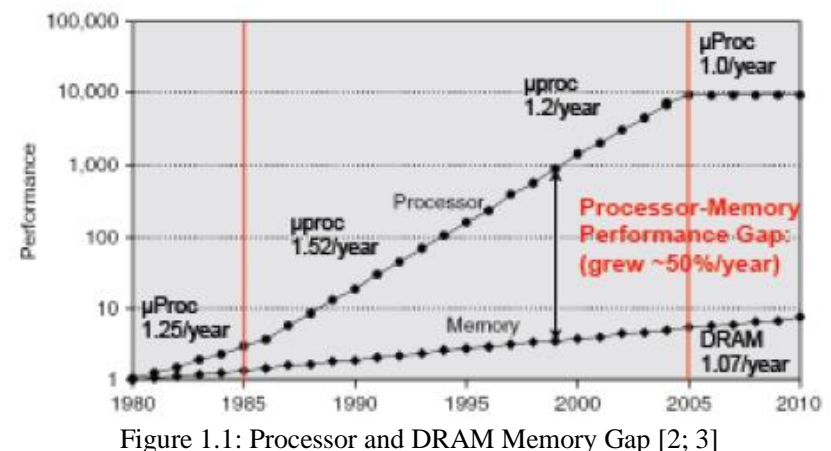

\section{DESIGNING SR AND JK-FLIP FLOPS}

We start by designing JK-FF from first principle Set and Reset Flip Flop (SR-FF) using NOR and NAND gate Configurations. When the 'forbidden states' of an SR-Flip Flop (as shown in Table 1.1) are converted to toggling states, 
a JK-Flip Flop is so obtained. Hence, such a JK-Flip Flop retains the rest features of an SR-Flip Flop, such as its resting and active states; thus making a JK-Flip Flop to attain $75 \%$ active states utilization as against $50 \%$ active states utilization of an SR-Flip Flop on conversion of SR-FF to JKFF. The remaining $25 \%$ rest state of SR-FF is being examined in this study.

\subsection{Design Logic Circuit of an SR-Flip Flop}

The Truth Table of Table 1.1 is converted into a K-Map in order to obtain the minimized logic equations of the SR-Flip Flop as depicted in Table 1.2.

\begin{tabular}{|c|c|}
\hline \multicolumn{2}{|c|}{ Table 1.2: K-Map of SR-FF from Table 1.1 of Chapter One } \\
\hline (SR-FF) Using NOR Gates & (SR-FF) Using NAND Gates \\
\hline \begin{tabular}{l|llll}
$Q_{n}$ & 00 & 01 & 11 & 10
\end{tabular} & \begin{tabular}{l|llll}
$Q_{n}$ & 00 & 01 & 11 & 10
\end{tabular} \\
\hline$0 \longdiv { 0 } / 0 / d x$ & 0 (ब) 1,00 \\
\hline 1100001 & 1 d 1010 \\
\hline $\begin{array}{c}Q_{n+1}=S+\bar{R} Q_{n} \ldots \ldots(1 a) \\
\bar{Q}_{n+1}=R+\bar{S}_{n \ldots \ldots}(1 b)\end{array}$ & $\begin{array}{l}Q_{n+1}=\bar{S}+R Q_{n} \ldots \ldots(1,1 a) \\
\bar{Q}_{n+1}=\bar{R}+S \bar{Q}_{n \ldots \ldots}(1,2 b)\end{array}$ \\
\hline
\end{tabular}

Logic equations $(1 \mathrm{a}, 1 \mathrm{~b}) \&(1.1 \mathrm{a}, 1.2 \mathrm{~b})$ are derived from the K-Map and they can be used to construct the Flip Flop using the different gates as given by the equations. However, it is customary to use NOR and NAND gates to construct logic equations (1a, 1b) and $(1.1 \mathrm{a}, 1.2 \mathrm{~b})$ respectively by converting the equations into these gates. The mathematical analysis of these equations as ascertained in [12] \& [13] is as follows:

$$
\frac{\boldsymbol{Q}_{\boldsymbol{n}+\boldsymbol{1}}=\boldsymbol{S}+\overline{\boldsymbol{R}} \boldsymbol{Q}_{\boldsymbol{n}} \ldots \ldots}{\overline{\bar{R} Q_{n}}}=\overline{\overline{R+\bar{Q}_{n}}} \ldots \ldots(1 c)
$$

Put equation (1c) into (1a), we have

$$
Q_{n+1}=S+\overline{R+\bar{Q}_{n}}
$$

Complement equation (1d), we have

$$
\begin{gathered}
\overline{\boldsymbol{Q}}_{\boldsymbol{n + 1}}=\overline{\boldsymbol{S}+\overline{\boldsymbol{R}+\overline{\boldsymbol{Q}}_{\boldsymbol{n}}}} \ldots \ldots(\mathbf{1} \boldsymbol{e}) \\
\overline{\boldsymbol{Q}}_{n+\mathbf{1}}=\boldsymbol{R}+\overline{\boldsymbol{S}}_{\overline{\boldsymbol{Q}}} \ldots \ldots(\mathbf{1} \boldsymbol{b}) \\
\overline{\overline{\bar{S} \bar{Q}_{n}}}=\overline{S+Q_{n}} \ldots \ldots(1 \mathrm{f})
\end{gathered}
$$

Put equations (1f) into (1b), we have

$$
\bar{Q}_{n+1}=R+\overline{S+Q_{n}} \ldots \ldots(1 g)
$$

Complement equation (1g), we have

$$
Q_{n+1}=\overline{R+\overline{S+Q_{n}}}
$$

Combining equations (1e) and (1h) results in the construction of SR-FF Logic Circuit Diagram using only NOR gates as shown in Figure 1.2(a). Similarly the same procedure is followed to obtain the logic circuit diagram of SR-FF using NAND Gates as shown in Figure 1.2(b).

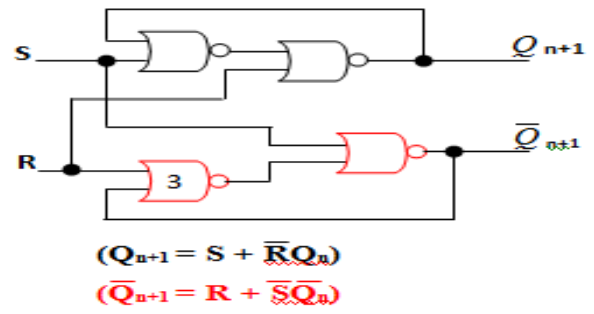

Fig. 1.2(a): SR NOR gates Flip Flop Equation Omotosho \& Ogunlere (2013)

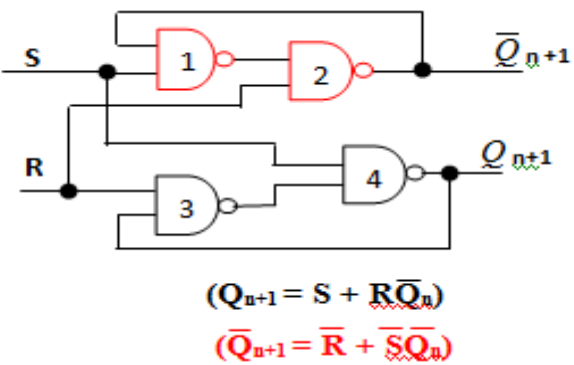

Fig. 1.2(b): SR NAND gates Flip Flop Equation Omotosho \& Ogunlere (2013)

\begin{tabular}{|c|c|c|c|c|c|c|c|c|c|}
\hline & \multicolumn{3}{|c|}{ JK-FF } & \multicolumn{6}{|c|}{ PROPOSED POSSIBLE FLIP FLOPS EXIENSION } \\
\hline $\mathrm{Li}$ & $\mathrm{J} \mid \mathrm{K}$ & $Q_{a} Q_{*}$ & Transition & Transi & & & & ble $($ t & puts, $Q_{n}$. \\
\hline 1 & 00 & \begin{tabular}{|l|l|l|l|l|}
0 & 0
\end{tabular} & Resting & ? $\} 12$ & & is state is requited to be converted into actir & 0 & 1 & 1 \\
\hline 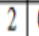 & \begin{tabular}{l|l|l|l|l|l|}
0 & 0
\end{tabular} & \begin{tabular}{|l|l|l|}
1 & 1 \\
\end{tabular} & Resting & $?+12$ & & is state is required to be converted into actis & 0 & 1 & 0 \\
\hline & 01 & 00 & Active & Active & & & & & \\
\hline 4 & 01 & 10 & Active & Active & & & $\mathrm{z}$ & & $\mathrm{z}$ \\
\hline & 10 & 01 & Active & Active ( & $5 \%$ & & $F$ & & 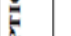 \\
\hline & 10 & 11 & Active & Active & & & $\overline{0}$ & & $\overline{0}$ \\
\hline & 11 & 01 & Toggle & Active & & his state is a dready attive by being togeglimg & & & \\
\hline & 11 & 10 & Toggle & Active & & his state is areedy active by beeng togeling. & & & \\
\hline
\end{tabular}

\section{PROPOSED FLIP FLOP EXTENSION}

Possibilities of alternative Flip Flops, to conventional JKFlip Flop are proposed. These different JK-Flip Flops are tagged as highlighted in Table 1.2, and are stated according to our adopted convention as follows:

Table 1.2: Proposed Flip Flop Extension Concept

Option 1: 87.5\% utilization Flip Flops identified as JKFF Extension - 0; (for Rest $1 \rightarrow 0$ ); One state at rest.

Option 2: 87.5\% utilization Flip Flops identified as JKFF Extension - 1; (for Rest $0 \rightarrow 1$ ); One state at rest.

\begin{tabular}{|c|c|c|c|c|c|c|c|c|c|c|}
\hline \multicolumn{11}{|c|}{ Table 1.3: Truth Table of Different JK-Flip Flops } \\
\hline K-MAP & \multicolumn{5}{|c|}{ OPTION 1} & \multicolumn{5}{|c|}{ OPTION 2} \\
\hline$J \overline{K Q_{n}=000 \text { (Option 1) }}$ & \multicolumn{5}{|c|}{$J K Q_{n}=000(87.5 \%)$} & \multicolumn{5}{|c|}{$J K Q_{n}=001(87.5 \%)$} \\
\hline $\begin{array}{lllll}Q_{n} & 00 & 01 & 11 & 10\end{array}$ & $\mathrm{~J}$ & K & $Q_{n}$ & $a_{p+1}$ & State & $n$ & $+1 \mathrm{~K}$ & $Q_{h}$ & $Q_{n+1}$ & States \\
\hline 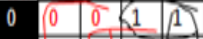 & 0 & 0 & 0 & 0 & Rest & 0 & 0 & & 1 & Active \\
\hline \begin{tabular}{ll|l|l|l|l}
1 & 0 & 0 & 0 & 1 \\
\end{tabular} & 0 & 0 & 17 & 0 & Active & 0 & 0 & 1 & 1 & Rest \\
\hline & 0 & 1 & 0 & & Active & 0 & 1 & 0 & 0 & Active \\
\hline \begin{tabular}{l|llll}
$Q_{2}$ & 00 & 01 & 11 & 10
\end{tabular} & 0 & 1 & $1-$ & & Active & 0 & 1 & $1 \rightarrow$ & 0 & Active \\
\hline \begin{tabular}{ll|l|l|l}
0 & 1 & 0 & 1 & 1 \\
\end{tabular} & 1 & 0 & $0 \overline{7}$ & & Active & 1 & 0 & $0=$ & 1 & Active \\
\hline 11001 & 1 & 0 & 1 & 1 & Active & 1 & 0 & & 1 & Active \\
\hline JKQ -001 (Option 2) & 1 & 1 & 07 & & Active & 1 & 1 & $0 \rightarrow$ & 1 & Active \\
\hline & 1 & 1 & 17 & & Active & 1 & 1 & $1 \rightarrow$ & क & Active \\
\hline
\end{tabular}

Option 3: 100\% utilization Flip Flops identified as XYFF - No Rest States.

The Truth Table of these possible options to conventional JK-Flip Flop tagged JK-FF Extensions is presented in Tables 1.3 and 1.4 with reference to Conventional JK-FF from where their simplified Boolean equations are derived. 


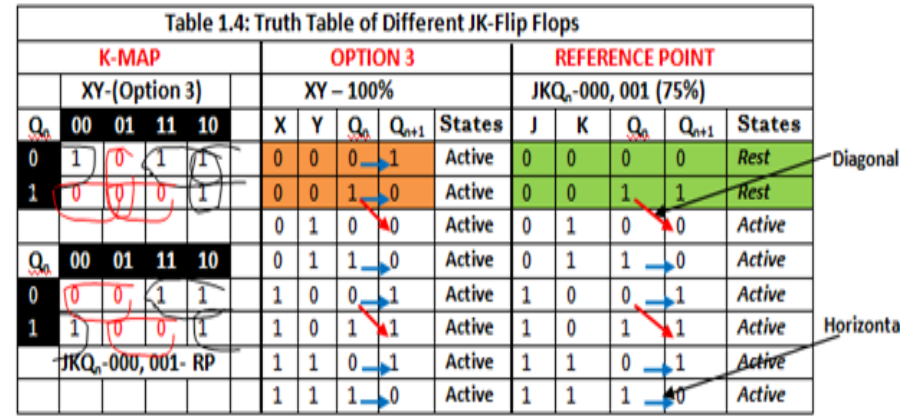

\section{MATHEMATICAL ANALYSIS OF JK-FF DESIGN}

The conversion of SR-FF to JK-FF as a Positive Logic Design is mathematically analyzed as follows using DeMorgan's theorem, Boolean algebra rules and K-Map technique.

\subsection{JK-000, 001 Rest: Conventional JK Flip Flop at 75\% active states utilization}

From K-map on JK Flip Flop at 75\% active states utilization, the simplified equation of 2.1 is derived using NOR gate configuration as Positive Logic design.

$$
Q_{n+1}=J \bar{Q}_{n}+\bar{K} Q_{n}
$$

NAND GATES $(1,2,3)$

$$
Q_{n+1}=\overline{\bar{J} \overline{\mathbf{Q}}_{n}+\bar{K} Q_{n}}=\overline{\bar{J} \overline{\boldsymbol{Q}}_{n} \cdot \overline{\bar{K} Q_{n}}}
$$

NOR GATES $(1,2,3)$

$$
\begin{gathered}
\overline{\overline{J \bar{Q}_{n}}}=\overline{\bar{J}+Q_{n}} . \\
\overline{\overline{\bar{K} Q_{n}}}=\overline{K+\bar{Q}_{n}}
\end{gathered}
$$

Substitute equations (2.1b) \& (2.1c) into equation (2.1), we have

$$
\begin{aligned}
& Q_{n+1}=\overline{\bar{J}+Q_{n}}+\overline{K+\bar{Q}_{n}} \\
& \bar{Q}_{n+1}=\overline{\overline{J+Q_{n}}}+\overline{K+\bar{Q}_{n}} \\
& \bar{Q}_{n+1}=K Q_{n}+\bar{J} \bar{Q}_{n}
\end{aligned}
$$

NAND GATES $(4,5,6)$

$$
\bar{Q}_{n+1}=\overline{\overline{K Q_{n}+\bar{J} \bar{Q}_{n}}}=\overline{\overline{K Q_{n}}} \cdot \overline{\bar{J} \bar{Q}_{n}}
$$

NOR GATES $(4,5,6)$

$$
\begin{gathered}
\overline{\overline{\bar{J} \bar{Q}_{n}}}=\overline{J+Q_{n}} . \\
\overline{\overline{K Q_{n}}}=\overline{\bar{K}+\bar{Q}_{n}}
\end{gathered}
$$

Substitute equations (2.2b) \& (2.2c) into equation (2.2), we have

$$
\begin{gathered}
\bar{Q}_{n+1}=\overline{\bar{K}+\bar{Q}_{n}}+\overline{J+Q_{n}} \\
\boldsymbol{Q}_{n+1}=\overline{\overline{\boldsymbol{K}+\overline{\boldsymbol{Q}}_{n}}+\overline{\boldsymbol{J}+\boldsymbol{Q}_{n}}}
\end{gathered}
$$

Combining equations (2.1a) and (2.2a) will produce NAND gate configuration of Figure 1.2a. Combining equations (2.1e) and (2.2e) will produce NOR gate configuration of Figure 1.2b.

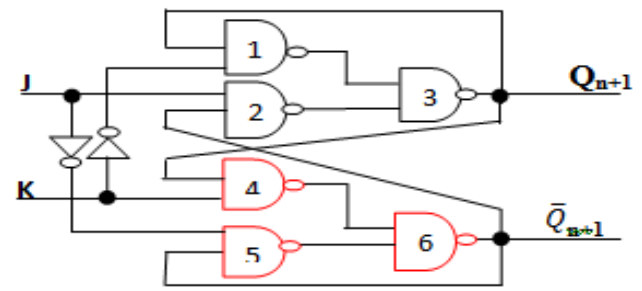

Figure 1.2 (a): Logic Circuit of Conventional JK-Flip Flop - 75\% (NAND Gate Configuration.)

Therefore, equations (2.1e) \& (2.2e) can be combined as presented in Figure 1.2b

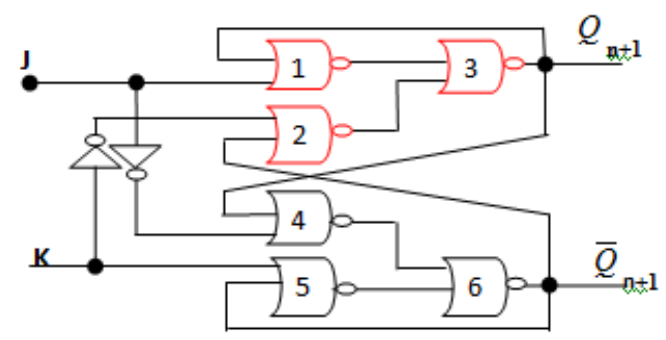

Figure 1.2 (b): Logic Circuit of Conventional JK-Flip Flop - 75\% (NOR Gate Configuration)

Note that the positive logic design configuration is actually the complement of the negative logic design configuration; which shows that either design will produce the same performance.

\section{USING CHARACTERISTIC EQUATIONS TO SIMPLIFIED JK-FLIP FLOP DESIGN}

The complete Logic Circuit Diagrams based on the K-map analysis and the characteristic equations derived from the state diagram Excitation Table are constructed in Figures $1.2(\mathrm{c})$ and $1.2(\mathrm{~d})$ respectively. These two circuit diagrams perform the same memory element functions.

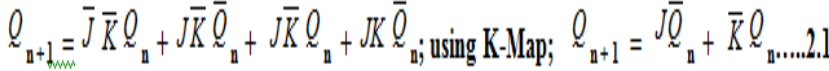

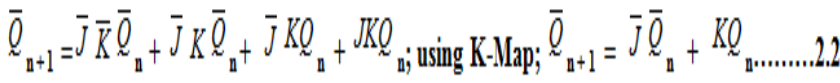

Equations 2.1 and 2.2 are used to produce the circuit diagram of Figure 1.2(d) which is the equivalent of circuit diagram in Figure 1.2(c). 


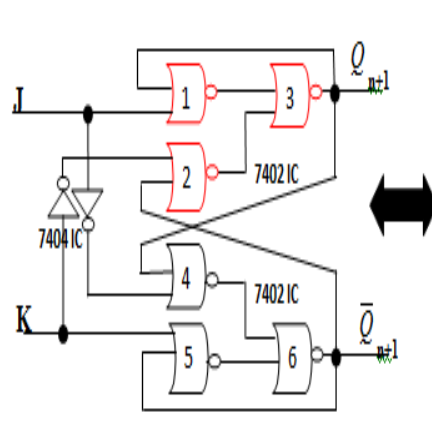

Figure 1.2(c)

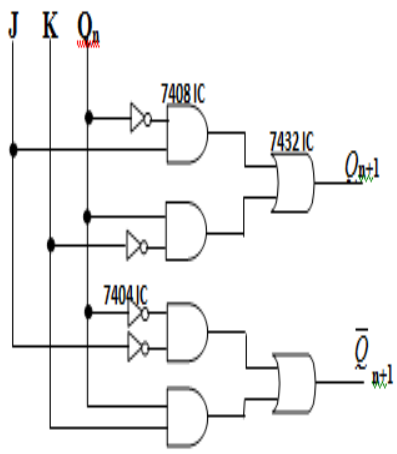

Figure 1.2(d)
Figure 1.2(c): NOR gates JK-FF based on K-map Figure 1.2(d): NOT, AND \& OR gates JK-FF based on characteristic equations

\section{OPTION 1: DESIGNING FLIP FLOP EXTENSION - 0 AT $87.5 \%$ (REST $1 \rightarrow 0$ )}

$$
Q_{n+1}=J \bar{K}+J \bar{Q}_{n}=J\left(\bar{K}+\bar{Q}_{n}\right)
$$

NAND GATES $(1,2,3)$

$$
Q_{n+1}=\overline{\overline{J \bar{K}+J \bar{Q}_{n}}}=\overline{\overline{\bar{J} \bar{K} \cdot \overline{J \bar{Q}_{n}}}}
$$

\section{NOR GATES}

$$
\begin{gathered}
\boldsymbol{Q}_{\boldsymbol{n}+\mathbf{1}}=\overline{\overline{\boldsymbol{J}\left(\overline{\boldsymbol{K}}+\overline{\boldsymbol{Q}}_{\boldsymbol{n}}\right)}}=\overline{\overline{\boldsymbol{J}}+\overline{\left(\overline{\boldsymbol{K}}+\overline{\boldsymbol{Q}}_{\boldsymbol{n}}\right)}} \\
\bar{Q}_{n+1}=\bar{J}+K Q_{n} \ldots \ldots \ldots \ldots
\end{gathered}
$$

\section{NAND GATES $(4,5)$}

$$
\bar{Q}_{n+1}=\overline{\overline{J+K Q_{n}}}=\overline{J \cdot \overline{K Q_{n}}}
$$

NOR GATES

$$
\overline{\overline{K Q_{n}}}=\overline{\bar{K}+\bar{Q}_{n}}
$$

Substitute equation (3.2b) into equation (3.2), we have

$$
\begin{gathered}
\bar{Q}_{n+1}=\bar{J}+\overline{\bar{K}+\bar{Q}_{n}} . \\
\boldsymbol{Q}_{n+1}=\overline{\overline{\boldsymbol{J}}+\overline{\overline{\boldsymbol{K}}+\overline{\boldsymbol{Q}}_{n}}} .
\end{gathered}
$$

Combining equations (3.1a) \& (3.2a) to produce NAND gate configuration of Figure 2.1a; while the combination of equations (3.1b) \& (3.2e) to produce NOR gate configuration is not feasible because equation $(3.1 \mathrm{~b})=$ equation (3.2e).

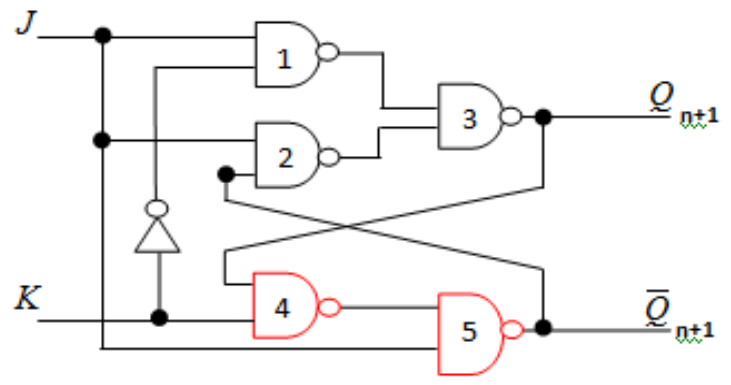

Figure 2.1(a): Logic Circuit of JK-FF Extension -0 at $87.5 \%$ (NAND Gate Configuration)

The complete Logic Circuit Diagrams based on the K-map analysis and the characteristic equations of 3.1 and 3.2 as derived from the state diagram excitation table are constructed in Figures 2.2(b) and 2.2(c) respectively. These two circuit diagrams perform the same memory element functions.

$$
\begin{aligned}
& Q_{n+1}=J \bar{K}_{n_{n}}+J \bar{K} Q_{n}+J K \bar{Q}_{n}=J \bar{Q}_{n}+J \bar{K}=J\left(\bar{K}_{+} \bar{Q}_{)}\right. \\
& \bar{Q}_{n+1}=\bar{J} \bar{K}_{Q_{n}+} \bar{J} \bar{K}_{n} Q_{n} \bar{J} K \bar{Q}_{n}+\bar{J} K Q_{n}+J K Q_{n}=\bar{J}_{+} K Q_{n}
\end{aligned}
$$

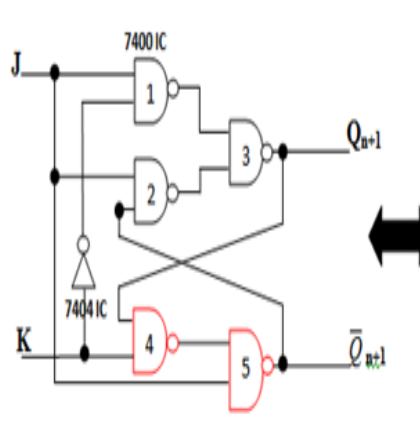

Figure 2.2(b)

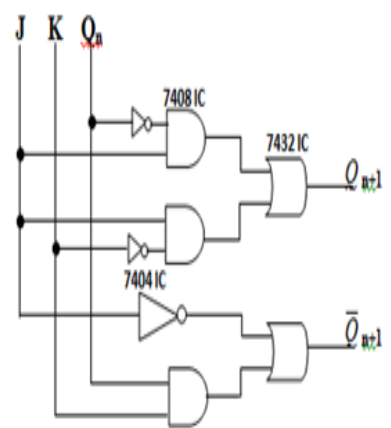

Figure 2.2(c)
Figure 2.2(b): JK-FF Extension-0 at $87.5 \%$ using NAND gates based on Kmap

Figure 2.2(c): JK-FF Extension-0 at 87.5\% using NOT, AND \& OR gates based on characteristic equations

\section{OPTION 2: DESIGNING FLIP FLOP EXTENSION - 1 AT $87.5 \%$ (REST $0 \rightarrow 1$ )}

$$
\begin{aligned}
& \bar{Q}_{n+1}=K \bar{J}+K Q_{n}=K\left(\bar{J}+Q_{n}\right) . \\
& \text { NAND }(1,2,3) \\
& \bar{Q}_{n+1}=\overline{\overline{K \bar{J}+K Q_{n}}}=\overline{\overline{K \bar{J}} \cdot \overline{K Q_{n}}} \\
& \text { NOR } \\
& \overline{\boldsymbol{Q}}_{n+1}=\overline{\overline{\boldsymbol{K}\left(\bar{J}+\boldsymbol{Q}_{n}\right)}}=\overline{\boldsymbol{K}+\overline{\left(\bar{J}+\boldsymbol{Q}_{n}\right)}} \\
& Q_{n+1}=\bar{K}+J \bar{Q}_{n}
\end{aligned}
$$

NAND $(4,5)$ 


$$
Q_{n+1}=\overline{\bar{K}+J \bar{Q}_{n}}=\overline{K \cdot \bar{J} \overline{\bar{Q}_{n}}}
$$

NOR

$$
\overline{\overline{J \bar{Q}_{n}}}=\overline{\bar{J}+Q_{n}}
$$

Substitute equation (4.2b) into equation (4.2), we have

$$
\begin{gathered}
\bar{Q}_{n+1}=\bar{K}+\overline{\bar{J}+Q_{n}} \\
\boldsymbol{Q}_{\boldsymbol{n}+\mathbf{1}}=\overline{\overline{\boldsymbol{K}}+\overline{\overline{\boldsymbol{J}}+\boldsymbol{Q}_{\boldsymbol{n}}}}
\end{gathered}
$$

Combining equations (4.2a) \& (4.1a) to produce NAND gate configuration of Figure 3.1a; while the combination of equations (4.1b) \& (4.2e) to produce NOR gate configuration is not feasible because equation $4.1 \mathrm{~b}=4.2 \mathrm{e}$.

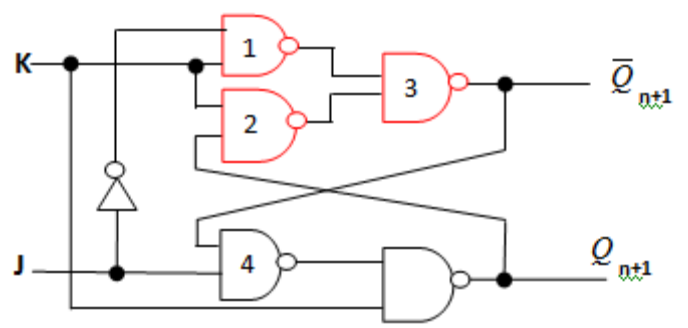

Figure 3.1(a): Logic Circuit of JK-FF Extension - 1 at $87.5 \%$ (NAND Gate Configuration)

The complete Logic Circuit Diagrams based on the K-map analysis and the characteristic equations of 4.2 and 4.1 as derived from the state diagram excitation Table are constructed in Figures 3.1(b) and 3.1(c) respectively. These two circuit diagrams perform the same memory element functions.

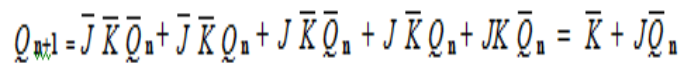

$$
\begin{aligned}
& \bar{Q}_{n+1}=\bar{J} K \bar{Q}_{\mathrm{n}}+\bar{J} K{\underset{\sim}{\mathrm{n}}}_{\mathrm{n}}+J Q_{\mathrm{n}}=\bar{J} K+K Q_{\mathrm{n}}=K\left(\bar{J}+Q_{\mathrm{n}}\right)
\end{aligned}
$$
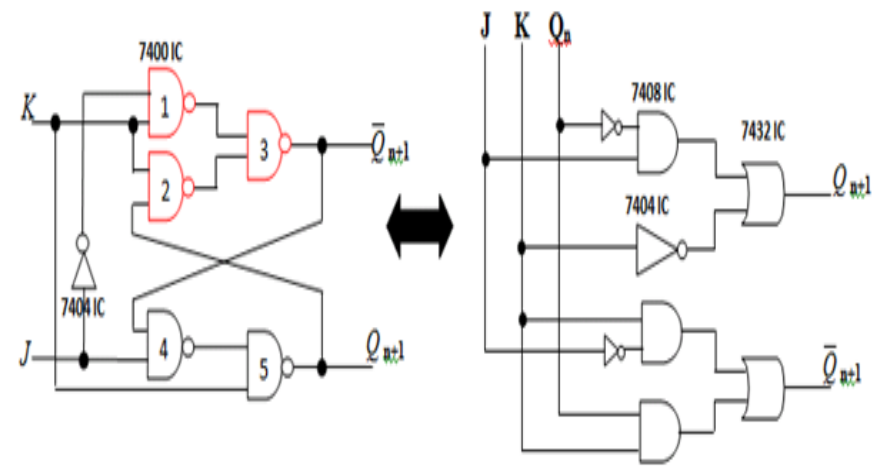

Figure 3.1(b)

Figure 3.1(c)

Figure 3.1(b): JK-FF Extension-1 at 87.5\% using gates NAND based on

$$
\text { K-map }
$$

Figure 3.1(c): JK-FF Extension-1 at 87.5\% using NOT, AND \& OR gates based on characteristic equations

\section{OPTION 3: FLIP FLOP EXTENSION; NO REST} STATES (100\%)- SUBSTITUTE $\mathrm{J}=\mathrm{X}$ AND $\mathrm{K}=\mathrm{Y}$

$$
\begin{aligned}
Q_{n+1}=X \bar{Y}+X \bar{Q}_{n}+\bar{Y} \bar{Q}_{n} \\
=X\left(\bar{Y}+\bar{Q}_{n}\right)+\bar{Y} \bar{Q}_{n}
\end{aligned}
$$

\section{NAND GATES $(1,2,3,4)$}

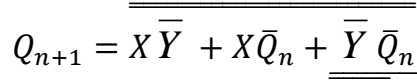

$$
\begin{aligned}
& =\overline{\overline{X \bar{Y}}} \cdot \overline{X \bar{Q}_{n}} \cdot \overline{\bar{Y} \bar{Q}_{n}} \\
& Q_{n+1}==\overline{\overline{X \bar{Y}} \cdot \overline{X \bar{Q}_{n}} \cdot \overline{\bar{Y}} \overline{\mathbf{Q}}_{n}}
\end{aligned}
$$

NORGATES $(4,6,7,8)$

Replace

$$
\begin{gathered}
\overline{\overline{\bar{Y} \bar{Q}_{n}}}=\overline{\bar{Y}+Q_{n}} \ldots \\
\overline{\overline{X \bar{Y}}}=\overline{\bar{X}+Y} \ldots \\
\overline{\overline{X \bar{Q}_{n}}}=\overline{\bar{X}+Q_{n}} .
\end{gathered}
$$

Substitute equations (5.1b), (5.1c) \& (5.1d) into equation (5.1a), we have

$$
\begin{aligned}
& Q_{n+1}=\overline{\bar{X}+Y}+\overline{\bar{X}+Q_{n}}+\overline{Y+Q_{n}} \\
& \overline{\boldsymbol{Q}}_{n+1}=\overline{\overline{\bar{X}+Y}}+\overline{\overline{\bar{X}}+Q_{n}}+\overline{\overline{Y+Q_{n}}} \\
& \bar{Q}_{n+1}=Y \bar{X}+Y Q_{n}+\bar{X} Q_{n} \\
& =Y\left(\bar{X}+Q_{n}\right)+\bar{X} Q_{n}
\end{aligned}
$$

NAND GATES $(5,6,7,8)$

$$
\begin{aligned}
& \bar{Q}_{n+1}=\overline{\overline{Y \bar{X}+Y Q_{n}+\bar{X} Q_{n}}} \\
& =\overline{\bar{Y} \bar{X}} \cdot \overline{Y Q_{n}} \cdot \overline{\bar{X} Q_{n}}
\end{aligned}
$$

\section{NOR GATES $(1,2,3,4)$}

Replace

$$
\begin{aligned}
\overline{\overline{Y \bar{X}}} & =\overline{\bar{Y}+X} . . \\
\overline{\overline{Y Q_{n}}} & =\overline{\bar{Y}+\bar{Q}_{n}} \\
\overline{\bar{X} Q_{n}} & =\overline{X+\bar{Q}_{n}}
\end{aligned}
$$

Substitute equations $(5.2 \mathrm{~b}),(5.2 \mathrm{c}) \&(5.2 \mathrm{~d})$ into equation (5.2), we have

$$
\begin{aligned}
\bar{Q}_{n+1} & =\overline{\bar{Y}+X}+\overline{\bar{Y}+\bar{Q}_{n}}+\overline{X+\bar{Q}_{n}} \\
Q_{n+1} & =\overline{\bar{Y}+X}+\overline{\bar{Y}+\bar{Q}_{n}}+\overline{X+\bar{Q}_{n}}
\end{aligned}
$$

Combining equations (5.1a) \& (5.2a) will produce NAND gate configuration of Figure $4.1 \mathrm{a}$; while combining 
equations (5.1f) \& (5.2f) will produce NOR gate configuration of Figure 4.1b

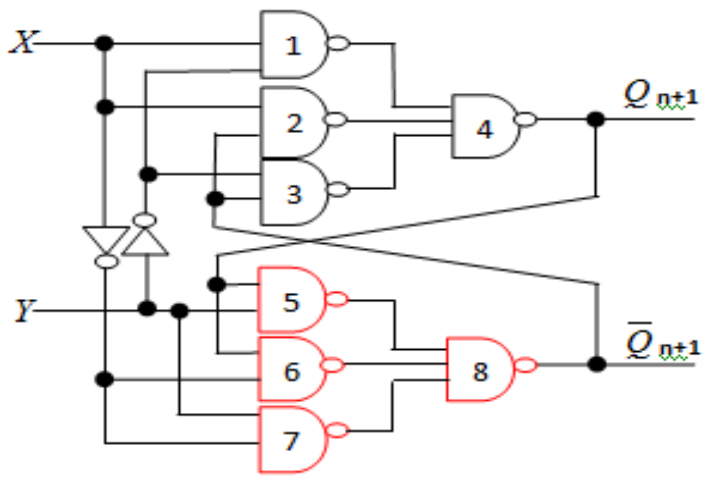

Figure 4.1(a): Logic Circuit of XY-FF No Rest at 100\% (NAND Gate Configuration)

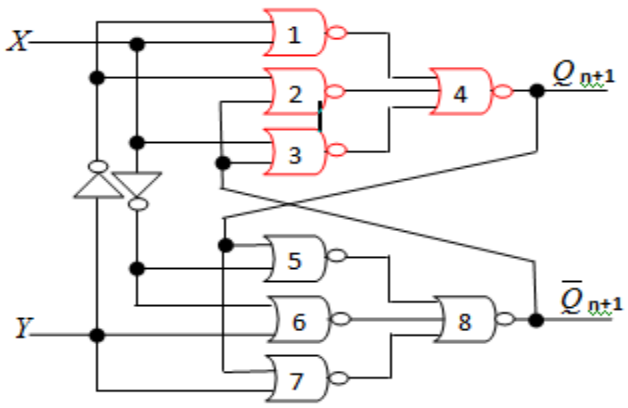

Figure 4.1(b): Logic Circuit of XY-FF No Rest at 100\% (NOR Gate Configuration)

The complete Logic Circuit Diagrams based on the K-map analysis and the characteristic equations of 5.1 and 5.2 as derived from the state diagram excitation Table are constructed in Figures 4.1(c) and 4.1(d) respectively. These two circuit diagrams perform the same memory element functions.

$$
\begin{aligned}
& Q_{n+1}=\bar{X} \bar{Y} \bar{Q}_{n}+X \bar{Y} \bar{Q}_{n}+X \bar{Y} \underline{\sim}_{n}+X Y \bar{Q}_{n}=X\left(\bar{Y}+\bar{Q}_{n}\right)+\bar{Y} \bar{Q}_{n} \\
& \bar{Q}_{n+1}=\bar{X} \bar{Y} Q_{n}+\bar{X} Y \bar{Q}_{n}+\bar{X} Y Q_{u}+X Y Q_{n}=Y\left(\bar{X}+Q_{n}\right)+\bar{X} Q_{n}
\end{aligned}
$$

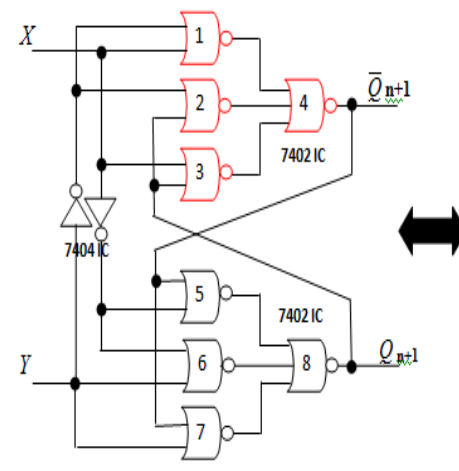

Figure 4.1 (c)

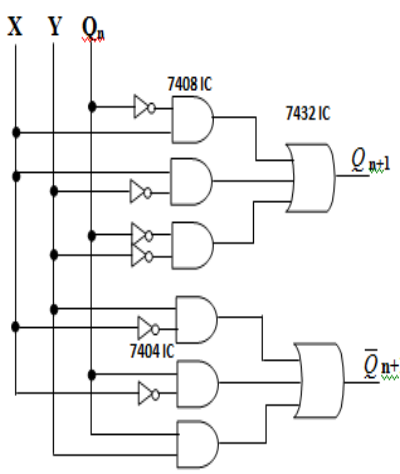

Figure 4.1 (d)
Figure 4.1(c): XY-FF Extension at 100\% using NOR gates based on Kmap

Figure 4.1(d): XY-FF Extension 100\% using NOT, AND \& OR gates based on characteristic equations

\section{SUMMARY OF THE VARIOUS FLIP FLOPS DESIGN}

Illustrated in Table 1.5 is the summary of the various Flip Flops design with the aim of verifying their gate structures

\begin{tabular}{|c|c|c|c|c|c|}
\hline \multicolumn{6}{|c|}{ Table 1.5: Review of the Different Flip Flops Design } \\
\hline \multirow[t]{2}{*}{$S / N$} & \multirow[t]{2}{*}{ TYPE OF FLIP FLOPS } & \multirow[t]{2}{*}{ CONFIGURATIONS } & \multicolumn{2}{|c|}{$\begin{array}{l}\text { NUMBER OF ACTIVE } \\
\text { TRANSITIONS/STATES }\end{array}$} & \multirow[t]{2}{*}{\begin{tabular}{|l} 
NUMBER OF \\
GATES \\
\end{tabular}} \\
\hline & & & Diagonal & Horizontal & \\
\hline 1. & JK-FF Two Resting States (75\%) & NANDorNORgates & 2 & 4 & 6 \\
\hline 2. & JK-FFExt-0 One Resting (87.5\%) & OnlyNANDgates & 2 & 5 & 5 \\
\hline 3. & JK-FFExt-10ne Resting (87.5\%) & OnlyNANDgates & 3 & 5 & 5 \\
\hline 4. & XY-No Rest(100\%) & NAND orNOR gates & 2 & 6 & 8 \\
\hline
\end{tabular}
and numbers.

\section{CONCLUSIONS}

Previous study revealed that very little research have been carried out on Flip Flops comparative analysis in the 100\%, $87.5 \%$ and $75 \%$ active states utilization on digital device applications. In this paper, a new approach of designing memory element (Flip Flop) with its active states utilization of $87.5 \%$ and or $100 \%$ as against the conventional Flip Flops at $50 \%$ and $75 \%$ active states utilization which will enhance memory performance have been developed. This is evidence in section 7 and 8 where the Flip Flop Extensions at $87.5 \%$ active states utilization is designed with one gate less than the conventional JK-Flip Flop. The uniqueness of this study is that computer memory speed performance can be enhanced through conventional JK-FF modification just as it is currently being done with its processor counterpart. This is a great advantage in performance over the conventional Flip Flops because fewer gates enhance performance (i.e., gate delay represents performance). The $87.5 \%$ Flip Flop extension memory cell is also portable (less transistors) and cheaper because it requires fewer transistors as against the conventional Flip Flops. An important issue in digital device design is that numbers of transistors represent hardware cost because in essence, maximizing performance and minimising cost in digital devices are part of the factors in seeking alternative design on more efficient and effective Flip flops.

Efforts should be geared towards verifying the effectiveness and efficiency of these newly design Flip Flops Extension over the existing conventional Flip Flops.

\section{REFERENCES}

O. J. Omotosho, Fundamentals of Digital Systems, Franco-Ola publishers, 2012

[2] Hennessy, J. L. \& David A. P., (2007), Computer Architecture: A Quantitative Approach. 4th ed., p. 289. Elsevier Inc.

[3] McCalpin, J. D., (2016), Memory Bandwidth and System Balance in HPC Systems, Invited Talk, International Conference for High Performance Computing, Networking, Storage, and Analysis. Available from: http://sites.utexas.edu/jdm4372/2016/11/22/sc16.

[4] C. Kim, Memory World in the Next Decade, Memory Division, Device Solution Network Business, Samsung, Semina 
presentation to Po-hang University of Science and Technology (POSTECH), Kyungpook, Korea,(2003

[5] O. J. Omotosho and S. O. Ogunlere, Design Analysis and Circuit Enhancements of SR-Flip Flop, International Journal of Engineering Sciences and research Technology (IJESRT), ISSN: $2277-9655,2013$

[6] O. J. Omotosho and S. O. Ogunlere, Analysis and Design of Different Flip Flops, Extension of Conventional JK-Flip Flops, International Journal of Engineering Sciences and research Technology (IJESRT), ISSN: 2277-9655, 2013 